\title{
Life Time Evolution of Wireless Sensor Network in Presence of Energy Hole at Sink
}

\author{
K. Anusha ${ }^{1}$, S. Srija ${ }^{2}$ \\ Assistant Professor, Computer Science Engg, Geethanjali College of Engineering and Technology, Hyderabad, India ${ }^{1,2}$
}

\begin{abstract}
Wireless sensor networks play vital role in data gathering. They often work in hostile environments where nodes are not having any energy refilling. Therefore the lifetimes of network and energy consumption are very important. The aim of any technique is to increase lifetime of the network with optimizations. The rationale behind this is that the network has sensor nodes that are resource constrained. In this paper we proposed a model used to determine the effects of energy hole and estimate network lifetime in presence of energy hole at sink. Energy hole is characterized and studied in order to find its effect on the lifetime of the network. We used NS2 simulations in order to evaluate our work. The experimental results revealed that the proposed model is useful to determine the life time of WSN.
\end{abstract}

Index Terms: Wireless Sensor Network (WSN), energy hole, routing, network lifetime, energy efficiency.

\section{INTRODUCTION}

Wireless Sensor Network (WSN) is a collection of sensor nodes that are located geographically in a distributed environment. The nodes in the network are capable of performing sensing. Typically they capture data from surroundings and send to base station or sink. The sensor nodes thus play vital role in many real world applications like temperature monitoring, surveillance, home monitoring, studying wildlife habitat besides serving other civilian and military purposes. A typical WSN appears as shown in Figure 1. The network can be connected to Internet servers and thus people from remote places can view data collected by sensor network. The problem with such devices in WSN is that they have limited energy with batter power. Once energy is completed, a sensor node gets switched off. Thus the lifetime of the network goes down drastically.

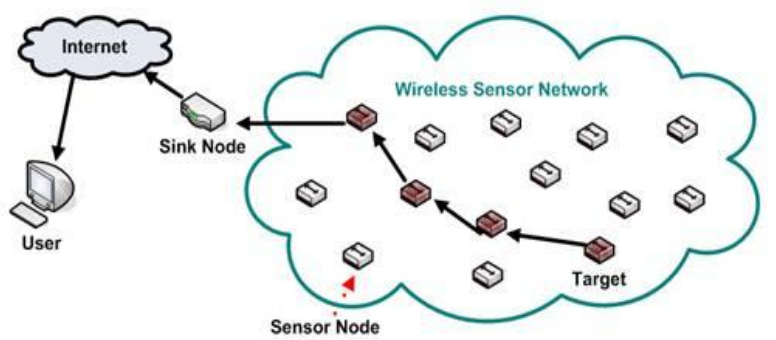

Figure 1: A typical WSN

As shown in Figure 1, the WSN needs to be supported with energy efficient means of communication in order to maximize network lifetime. There are many utilities when network lifetime is monitored and increased. Network lifetime is very important for continued services rendered by deployed WSN. In this context, it is essential to monitor the network for energy efficient approaches and optimizations. In this paper we proposed an analytical model that focuses on estimating network life time and energy efficiency in order to make well informed decisions. We proposed two algorithms towards this end. We built WSN using NS2 and the proof of the concept is demonstrated. The results revealed that the propoed model is capable of shedding light into the need for evaluating network life time and evolution of energy hole. As energy holes drain the energy of WSN, this study assumes significance. The remainder of the paper is structured as follows. Section II reviews related works. Section III provides details of the proposed system. Section IV provides experimental results. Section V provides conclusions. It also gives directions for future work.

\section{RELATED WORKS}

Many energy consumptions models in WSN came into existence for improving energy efficiency [1]. Data gathering networks like WSN consumes energy. However, energy efficiency is very important for energy constrained sensor nodes. Many approaches came into existence to have energy efficiency and improve network life time. Network life time analysis was done by many researchers [2], [3], and [4] where the focus was from network initialization to the time the death of fist node in the network. It is known as first node died time (FNDT). Distributed Energy Balancing Routing (DEBR) is an algorithm proposed in [5] for increasing FNDT. Network life time and cost models are explored in [6] for estimating node dynamics and network longevity. FNDT was explored in [7] in a WSN where clusters are used with spatial correlation. They used routing protocol to improve energy efficiency. The concept of upper bound derivation for FNDT is derived in [8] in cluster based WSN for efficient communications.

In [9] FNDT is highlighted as an important problem that needs to be taken care of for longevity of WSN. It is an 
important measure of performance of network as well. Annuli-based analytical model was proposed in [10] for finding network life time while aging process was proposed in [11] for the same. The network lifetime is the period between network initialization time and death of any sensor node for the first time. An improved model was proposed in [12] with respect to FNDT and optimal network lifetime. Event driven WSN was studied in [13] and [14] for monitoring entire lifetime of WSN.

Though many approaches came into existence to analyze network lifetime, there are few insights in literature on the energy hole and its effect on the network lifetime. Considering energy hole is very important for the research in WSN for network life longevity as explored in [15] and [16]. Many load balancing techniques are found in [17] for handling energy hole problem in WSN. They also proposed a heuristic algorithm that is distributed in nature. The problem also studied in [18], [19], [20] and [21] and stated that energy hole is located at the sink and energy efficient routing protocols can mitigate the effect of energy hold in order to improve the lifetime of WSN.

\section{PROPOSED APPROACH FOR LIFE TIME EVOLUTION}

In this paper, we proposed two algorithms for finding network life time and dealing with energy hole. At different stages of network, traffic load, energy consumption and lifetime of sensor are computed. This is done by the algorithm 1 .

Input: Network radius $\mathrm{R}$, transmission radius $\mathrm{r}$, node density of the network $\rho$, and other parameters.

Output: For each stage i and each node j, return the nodal traffic $\operatorname{loadP}_{j}^{(i)}$, energy consumptione ${ }_{j}^{(i)}$, as well as the energy transfer function $\mathrm{f}$ and lifetime vector 1 .

1. According to theorem 1 and 2 we have to calculate traffic load and energy consumption of all nodes at first stage $(\mathrm{i}=0)$ like $\left[\mathrm{P}_{1}^{0}, \mathrm{P}_{2}^{0}, \mathrm{P}_{3}^{0}, \mathrm{P}_{\mathrm{j}}^{0}, \ldots . . \mathrm{P}_{\mathrm{n}}^{0}\right],\left[\mathrm{e}_{1}^{0}, \mathrm{e}_{2}^{0}\right.$ $\left., e_{3}^{0}, e_{j}^{0}, \ldots . . e_{n}^{0}\right]$

2.then move onto the next stage i.e $\mathrm{i}=1$

3. while $(\mathrm{i}<\mathrm{n})\{$

Calculate life timel ${ }^{\mathrm{i}-1}$, ith batch of dead node region [ui, ui $+\varepsilon]$.

Calculate traffic load and energy consumption like [ $\mathrm{P}_{1}^{\mathrm{i}}$ $\left., P_{2}^{i}, P_{3}^{i}, P_{j}^{i}, \ldots . P_{n}^{i}\right],\left[e_{1}^{i}, e_{2}^{i}, e_{3}^{i}, e_{j}^{i}, \ldots . . e_{n}^{i}\right]$

Using theorem 3 and 4 .

i++

\}

Algorithm 1. At each network stage determine the traffic load, energy consumption and lifetime of sensor nodes.

Input: Network radius $\mathrm{R}$, transmission radius $\mathrm{r}$, node density of the network $\rho$, and other parameters.

Output: The energy hole boundary $\left[\mathrm{d}_{\text {shole }}, \mathrm{d}_{\text {ehole }}\right]$ and emerging time $t_{h}$.

1.Iterate first algorithm until we satisfy the below condition

while $(\mathrm{d}>=\mathrm{r})$

2.At that point we have to calculate the dead region boundary, emerging time.

3. Return values.

Algorithm 2. Determining the emerging time and boundary of the energy hole

Algorithm 2 proposed here determines the boundary of the energy hole and emerging time. The algorithm thus helps in energy whole evolution and measuring the dynamics of network life time.

\section{IV.EXPERIMENTAL RESULTS}

We used NS2 simulations to demonstrate proof of the concept. Experiments are made in terms of observations on network lifetime with different network size, transmission radius, number of sensor nodes, with different algorithms.

Table 1: Shows simulation environment

\begin{tabular}{|l|l|}
\hline PARAMETER & SPECIFICATION \\
\hline Simulation tools used & NS2 Network Simulator (ns-2.35) \\
\hline Simulation time & $10 \mathrm{sec}, 20 \mathrm{sec}, 30 \mathrm{sec}$ \\
\hline Number of nodes & $10,20,30,40$ \\
\hline Transmission range & $250 \mathrm{~m}$ \\
\hline Maximum speed & $0-20 \mathrm{~m} / \mathrm{sec}$ \\
\hline Application traffic & CBR [constant bit rate] [20] \\
\hline Packet size & 512 bytes \\
\hline Node mobility model & 10 packets/sec \\
\hline Protocol & AODV \\
\hline Number of runs & 36 \\
\hline
\end{tabular}

As shown in Table 1, it is evident that the simulation environment used for experiments is presented. It includes the protocol, simulation time, nodes, NS2 version, packet size and so on.

Table 2: ANDT with different network size

\begin{tabular}{|l|l|l|l|}
\hline \multirow{2}{*}{$\begin{array}{l}\text { Networ } \\
\text { k Size }\end{array}$} & \multicolumn{4}{|l|}{ Andt $(* \mathbf{1 0 0}$ rounds) } \\
\cline { 2 - 4 } & Simulation & $\begin{array}{l}\text { Our } \\
\text { Analysis }\end{array}$ & $\begin{array}{l}\text { Analysis } \\
\text { Of [17] }\end{array}$ \\
\hline 400 & 13 & 10 & 7 \\
\hline 600 & 14 & 11 & 7.5 \\
\hline 800 & 16 & 12.5 & 8 \\
\hline
\end{tabular}

As shown in Table 2, it is evident that the network size has its influence on the ANDT. The results show the difference among the analysis of [17], proposed analysis and simulation. 


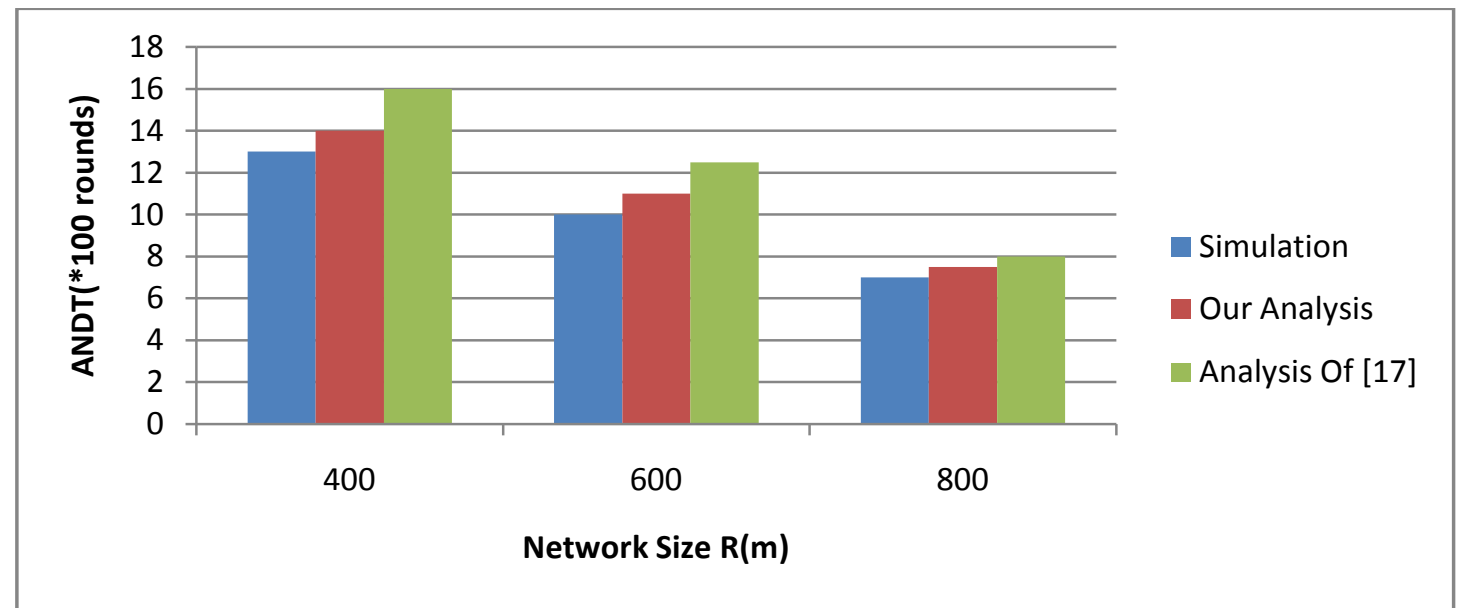

Figure 2: Network size vs. ANDT

As shown in Figure 2, the network size is taken in horizontal axis and ANDT is represented in vertical axis. The results revealed that ANDT has its influence on the size of the network.

Table 3: Transmission radius and network life time

\begin{tabular}{|l|l|l|l|l|l|l|l|l|l|l|l|l|}
\hline Transmission Radius r(m) & 50 & 60 & 70 & 80 & 90 & 100 & 110 & 120 & 130 & 140 & 150 & 160 \\
\hline Fndt In a & 0.5 & 0.5 & 0.6 & 0.6 & 0.7 & 0.8 & 0.8 & 0.8 & 0.9 & 0.9 & 0.9 & 0.9 \\
\hline 3\% Nodes Die In a & 0.5 & 0.6 & 0.7 & 0.7 & 0.7 & 0.8 & 0.8 & 0.9 & 0.9 & 1 & 1 & 1 \\
\hline Andt In a & 1 & 1.1 & 1.2 & 1.5 & 1.7 & 1.8 & 1.7 & 2.4 & 2.5 & 2.3 & 2 & \\
\hline Fndt In b & 1.3 & 1.5 & 2 & 2.3 & 3 & 3.5 & 3.6 & 4.5 & 4.5 & 5 & 5.3 & 5 \\
\hline
\end{tabular}

As shown in Table 3, it is evident that the transmission radius is considered from 50 through 160 incremented by 10 . The transmission radius has its impact on the FNDT and ANDT dynamics in WSN.

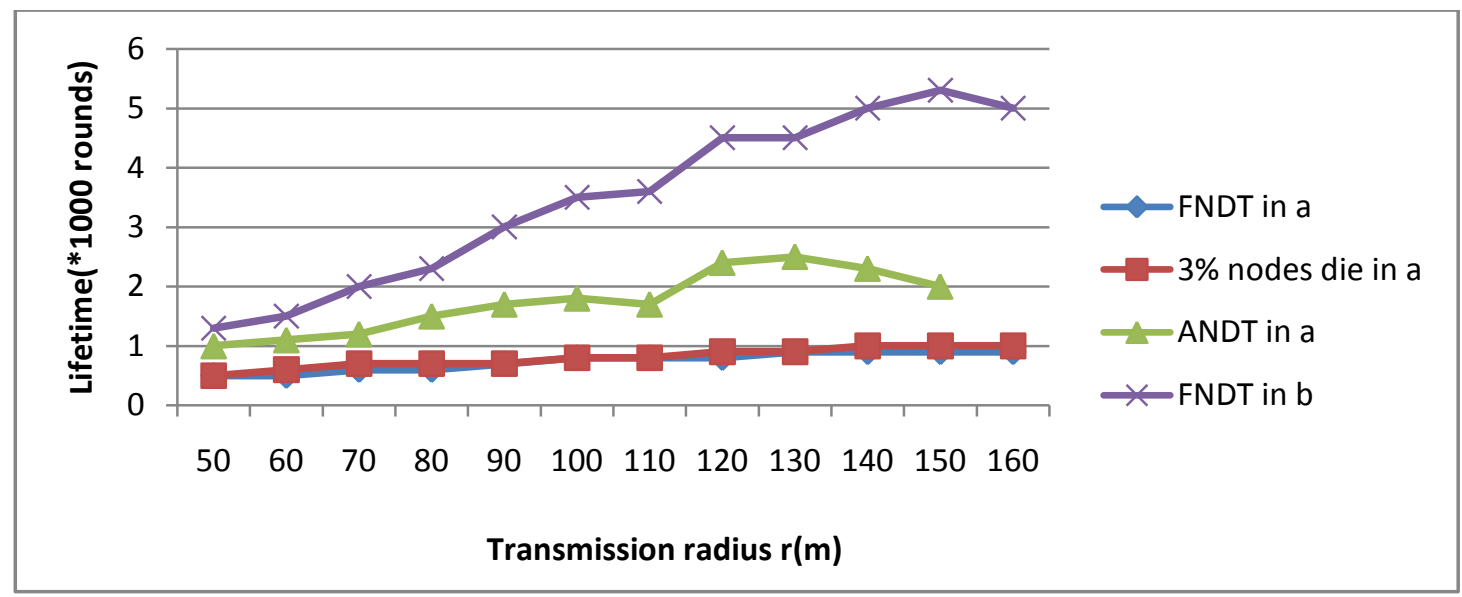

Figure 3: Transmission radius vs. network life time

As shown in Figure 3, it is evident that the network lifetime is measured in terms of FNDT and ANDT. The results revealed that the transmission radius has its impact on the lifetime of the network both with ANDT and FNDT models.

Table 4: Number of sensor nodes vs. network life time

\begin{tabular}{|l|l|l|l|l|l|l|l|}
\hline Number Of Sensor Nodes & 600 & 800 & 1000 & 1200 & 1400 & 1600 & 1800 \\
\hline Fndt,r=70m & 7.6 & 7.4 & 7 & 7.3 & 7.4 & 7.3 & 7.5 \\
\hline Fndt,r=90m & 8.5 & 8.5 & 8.5 & 9 & 8.5 & 8.7 & 8.6 \\
\hline Fndt,r=120m & 9.5 & 9.6 & 9.5 & 9.5 & 9.5 & 9.5 & 9.5 \\
\hline Andt,r=70m & 9.7 & 10 & 10 & 9.7 & 9.7 & 9.7 & 9.7 \\
\hline
\end{tabular}




\begin{tabular}{|l|l|l|l|l|l|l|l|}
\hline Andt,r=90m & 11 & 10.6 & 10.3 & 10.5 & 10.3 & 10.7 & 10.7 \\
\hline Andt,r=120m & 12 & 12 & 12 & 11.6 & 11.8 & 11.5 & 11.5 \\
\hline
\end{tabular}

As shown in Figure 4, the experiments are made with As shown in Figure 5, it is evident that different network different number of nodes. For each experiment the details sizes are considered and ANDT is plotted for different of ANDT and FNDT are recorded. algorithms like DCFR, DEBR and GGR.

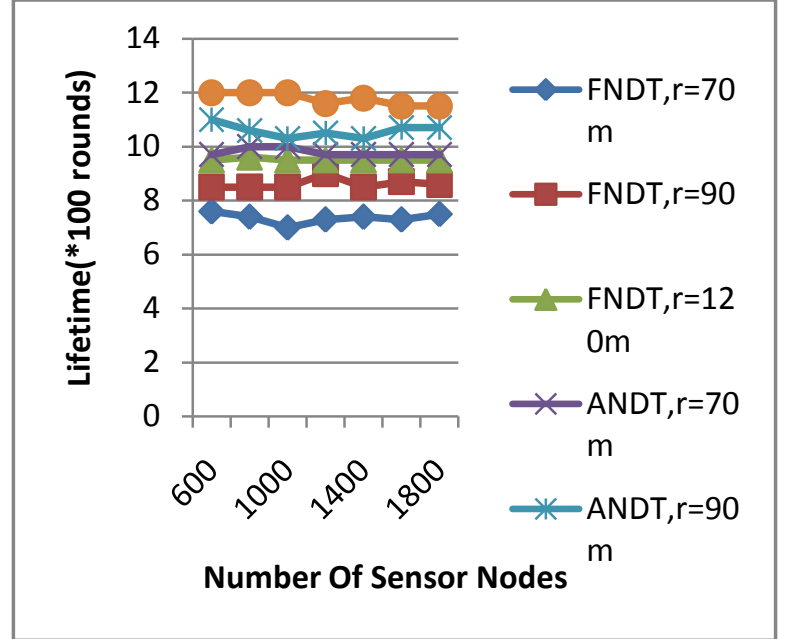

Figure 4: Number of sensor nodes vs. ANDT and FNDT

As presented in Figure 4, the results revealed that the number of sensor nodes have their impact on the network life time both in terms of ANDT and FNDT.

Table 5: Network size vs. ANDT

\begin{tabular}{|l|l|l|l|}
\hline \multirow{2}{*}{ Network SizeR(m) } & \multicolumn{3}{|c|}{ Andt(* 1000 rounds) } \\
\cline { 2 - 4 } & GGR & DEBR & DCFR \\
\hline 200 & 3.9 & 4.4 & 4.6 \\
\hline 300 & 2.5 & 3 & 3.3 \\
\hline 400 & 1.5 & 2.3 & 2.6 \\
\hline 500 & 1 & 1.7 & 2 \\
\hline
\end{tabular}

As shown in Table 5, the results of different mechanisms like GGR, DEBR and DCFR are presented in terms of ANDT in the presence of different network sizes.

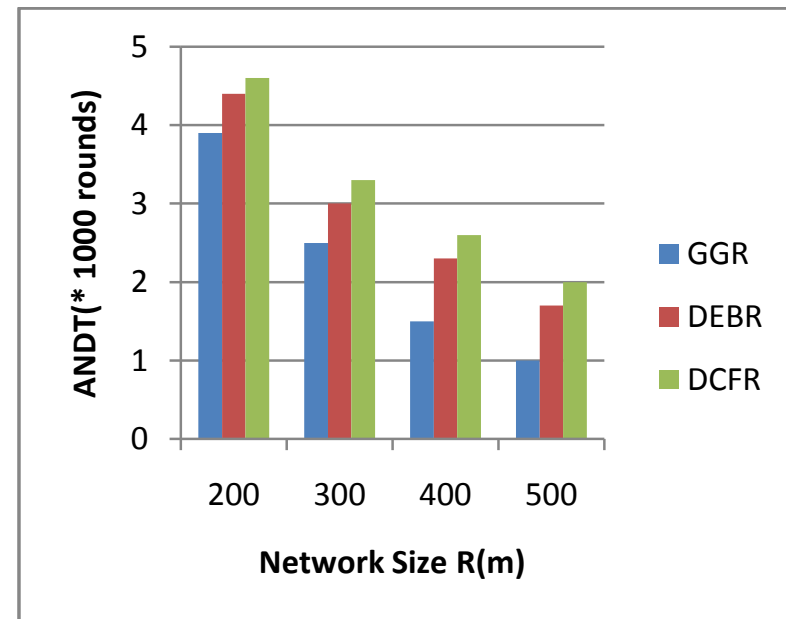

Figure 5: Network size vs. ANDT for different approaches
Table 6: Number of sensor nodes vs. GGR and DEBR performance with ANDT

\begin{tabular}{|l|l|l|l|l|l|}
\hline $\begin{array}{l}\text { Number Of Sensor } \\
\text { Nodes }\end{array}$ & 600 & 800 & 1000 & 1200 & 1400 \\
\hline GGR & 15 & 16 & 17 & 18 & 19 \\
\hline DEBR & 25 & 27 & 28 & 29 & 30 \\
\hline
\end{tabular}

As shown in Table 6, it is evident that the number of sensor nodes has its influence on ANDT with different algorithms such as GGR and DEBR.

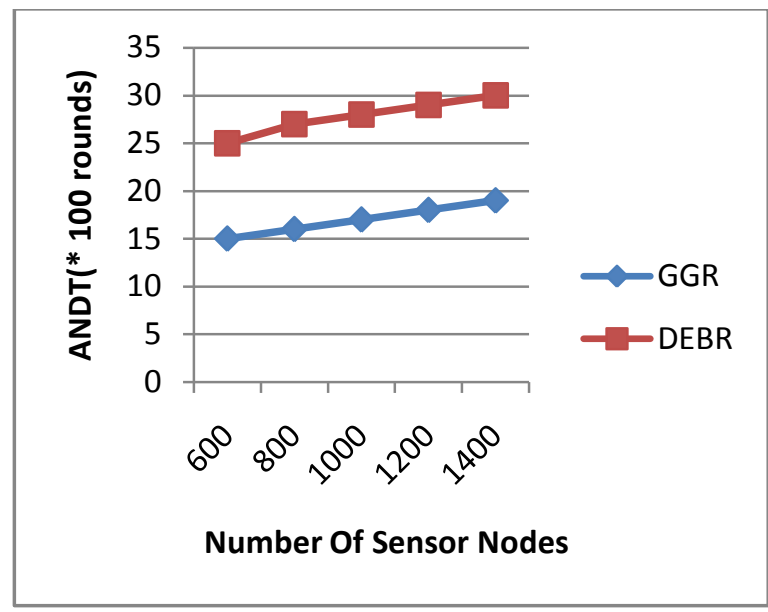

Figure 6: Number of sensor nodes vs. ANDT

As shown in Figure 6, the number of sensor nodes is presented in horizontal axis while the vertical axis shows ANDT measure. The results reveal that DEBR showed more ANDT when compared with that of GGR.

\section{V.CONCLUSIONS AND FUTURE WORK}

In this paper, we built an analytical model that helps in estimation of lifetime of WSN, energy consumption, and traffic load. It also finds evolution of network lifetime in presence of energy hole at sink node. The percentage of dead nodes is considered for computing network life time. It also considered network characteristic to leverage design and optimization of the proposed approach. Two algorithms are defined two achieve this. NS2 simulations are made to demonstrate proof of the concept. Our results show that the proposed model was able to estimate network life time, and energy whole evolution with minimal error rate. The results of analysis are applied to routing in WSN in order to improve the lifetime of network with energy harvesting. In future we improve our model with stochastic approaches. 


\section{REFERENCES}

[1] J. Ren, Y. Zhang, and K. Liu, "An energy-efficient cyclic diversionary routing strategy against global eavesdroppers in wireless sensor networks," Int. J. Distrib. Sensor Netw., vol. 2013, pp. 1-16, 2013.

[2] Y. Chen and Q. Zhao, "On the lifetime of wireless sensor networks," IEEE Commun. Lett., vol. 9, no. 11, pp. 976-978, Nov. 2005.

[3] J. Li and G. AlRegib, "Network lifetime maximization for estimation in multihop wireless sensor networks," IEEE Trans. Signal Process., vol. 57, no. 7, pp. 2456-2466, Jul. 2009.

[4] Q. Chen, S. Kanhere, and M. Hassan, "Analysis of per-node traffic load in multi-hop wireless sensor networks," IEEE Trans. Wireless Commun., vol. 8, no. 2, pp. 958-967, Feb. 2009.

[5] C.-S. Ok, S. Lee, P. Mitra, and S. Kumara, "Distributed energy balanced routing for wireless sensor networks," Comput. Ind. Eng., vol. 57, no. 1, pp. 125-135, 2009.

[6] Z. Cheng, M. Perillo, and W. Heinzelman, "General network lifetime and cost models for evaluating sensor network deployment strategies," IEEE Trans. Mobile Comput., vol. 7, no. 4, pp. 484497, Apr. 2008.

[7] A. Liu, X. Wu, Z. Chen, and W. Gui, "Research on the energy hole problem based on unequal cluster-radius for wireless sensor networks," Comput. Commun., vol. 33, no. 3, pp. 302-321, 2010

[8] S. Lee and H. Lee, "Analysis of network lifetime in cluster-based sensor networks," IEEE Commun. Lett., vol. 14, no. 10, pp. 900902, Oct. 2010.

[9] A. Ozgovde and C. Ersoy, "WCOT: A utility based lifetime metric for wireless sensor networks," Comput. Commun., vol. 32, no. 2 , pp. 409-418, 2009.

[10] K. Li, "Optimal number of annuli for maximizing the lifetime of sensor networks," J. Parallel Distrib. Comput., vol. 74, no. 1, pp. 1719-1729, 2014.

[11] J. Lee, B. Krishnamachari, and C. Kuo, "Aging analysis in largescale wireless sensor networks," Ad Hoc Netw., vol. 6, no. 7, pp. 1117-1133, 2008

[12] A. Liu, X. Jin, G. Cui, and Z. Chen, "Deployment guidelines for achieving maximum lifetime and avoiding energy holes in sensor network," Inf. Sci., vol. 230, pp. 197-226, 2013.

[13] H. Jaleel, A. Rahmani, and M. Egerstedt, "Probabilistic lifetime maximization of sensor networks," IEEE Trans. Autom. Control, vol. 58, no. 2, pp. 534-539, Feb. 2013.

[14] L. Zhang, S. Chen, Y. Jian, Y. Fang, and Z. Mo, "Maximizing lifetime vector in wireless sensor networks," IEEE/ACM Trans. Netw., vol. 21, no. 4, pp. 1187-1200, Aug. 2013.

[15] M. Perillo, Z. Cheng, and W. Heinzelman, "On the problem of unbalanced load distribution in wireless sensor networks," in Proc. IEEE GlobeCom Workshops, 2004, pp. 74-79.

[16] S. Olariu and I. Stojmenovic, "Design guidelines for maximizing lifetime and avoiding energy holes in sensor networks with uniform distribution and uniform reporting," in Proc. IEEE Int. Conf. Comput. Commun. (INFOCOM), 2006, pp. 1-12.

[17] R. Kacimi, R. Dhaou, and A. Beylot, "Load balancing techniques for lifetime maximizing in wireless sensor networks," Ad Hoc Netw., vol. 11, no. 8, pp. 2172-2186, 2013.

[18] A. Liu, D. Zhang, P. Zhang, G. Cui, and Z. Chen, "On mitigating hotspots to maximize network lifetime in multi-hop wireless sensor network with guaranteed transport delay and reliability," P2P Netw. Appl., vol. 7, no. 3, pp. 255-273, 2014.

[19] R. Rout and K. Ghosh, "Enhancement of lifetime using duty cycle and network coding in wireless sensor networks," IEEE Trans. Wireless Commun., vol. 12, no. 2, pp. 656-667, Feb. 2013.

[20] G. Chen, C. Li, M. Ye, and J. Wu, "An unequal cluster-based routing protocol in wireless sensor networks," Wireless Netw., vol. 15, pp. 193-207, 2009.

[21] S. Soro and W. Heinzelman, "Prolonging the lifetime of wireless sensor networks via unequal clustering," in Proc. IEEE Int. Parallel Distrib. Process. Symp., 2005, pp. 8-15. 